\title{
Energy Aware Scheduling and Queue Management for Next Generation Wi-Fi Routers
}

\author{
Husnu S. Narman and Mohammed Atiquzzaman \\ School of Computer Science, University of Oklahoma, Norman, OK 73019 \\ Email: \{husnu, atiq\}@ou.edu
}

\begin{abstract}
An increasing number of internet connected devices access large multimedia files over the Internet. Therefore, the bandwidth demand is exponentially increasing. To satisfy users' demands, multi-band wireless routers, which simultaneously support several bands, are developed. However, current multiband routers do not attempt to maximize the utilization of frequency bands nor consider the energy consumption. There are a limited number of previous works on multi-band wireless routers and those works do not address the energy efficiency and utilization problems of multi-band routers. Therefore, we have proposed an energy aware scheduling algorithm to ensure maximize utilization of multi-band routers while decreasing energy consumption through band sharing. Results show that the proposed method uses system resources efficiently and decreases energy consumption of the multi-band systems up to $60 \%$. Our proposed scheduling algorithm and related analysis will help network engineers build next generation wireless routers by considering performance metrics such as throughput and energy usage of multi-band Wi-Fi routers.
\end{abstract}

Index Terms-Multi-band, scheduling algorithm, queuing system, performance analysis, energy

\section{INTRODUCTION}

Increasing number of internet connected devices access large multimedia files (such as high definition audio, video, images, etc.) over the Internet. Therefore, the bandwidth demand is exponentially increasing [1]. To satisfy users' demands, multi-band wireless routers (MWRs) are developed. Current MWRs simultaneously support 2.4 and $5 \mathrm{GHz}$. It is expected that IEEE 802.11ad (WiGig) tri-band enabled devices transfer data rates upto $7 \mathrm{Gbps}$ by operating in the 2.4, 5 and $60 \mathrm{GHz}$ bands [2]. The benefit of using MWRs is to have less interference, higher capacity and better reliability. Exploitation of rarely-used frequency bands in wireless networks reduces interference in heavily-used frequency band, e.g., 2.4 $\mathrm{GHz}$, thereby increasing total capacity of the wireless network.

Current simultaneous MWRs use two different bands (2.4 $\mathrm{GHz}$ and $5 \mathrm{GHz}$ ) for different types of devices in a home network. However, they do not attempt to exploit the underutilized frequency band while other one is flooded with data. Moreover, using multi-band increases energy consumption because of multiple active bands, thus the number of active bands and their energy usages must be taken into account not to waste power. However, the current MWRs neither consider utilization of bands nor energy efficiency. Therefore, it is essential to propose an appropriate scheduling and queue management scheme for MWRs to ensure maximum possible utilization of the system resources [3], [4] by decreasing energy consumption. Hence, the aim of this work is to improve the energy efficiency of multi-band Wi-Fi routers while increasing utilization.

There have been several research works [1]-[3], [5], [6] reported in the literature. Verma and Lee [6] explain possible Wi-Fi architecture with multiple physical and link layers to simultaneously support multiple frequency bands. Singh et al. [1] proposed a method to assign different frequency bands to end-devices based on their distances from the access router. In [2], [5], authors proposed the use of $60 \mathrm{GHz}$ frequency band (having low range) to attain faster data transfer rate in wireless networks. However, none of these works [1]-[3], [5], [6] propose any scheduling algorithm for multi-band Wi-Fi routers to increase energy efficiency.

To the best our knowledge, there has been no earlier works on scheduling and queue management for multi-band wireless routers that attempts to maximize utilization of available bands. Moreover, our primary reports [7], [8] are the only previous works exist that proposed the sharing of multiple bands to transmit different classes of traffic. In [7], multishared-band router architecture is analyzed and the benefits of the multi-shared-band router architecture over the current multi-band router architecture are presented. In [8], the benefits of the multi-shared-band router architecture with different allocation policies over the single-band router architecture are demonstrated through an extensive simulation. However, energy aware scheduling algorithm in MWRs is not considered in our primary reports [7], [8]. However, it is essential to consider energy efficiency in the next generation MWRs because even standby energy billing cost of a single band WiFi router is $\$ 27$ (which is the highest standby cost in the home gadgets) per year according to Ecotricity [9]. This is a novel work that aims at attaining maximum possible band utilization while decreasing energy consumption.

The objective of this work is to ensure maximize utilization of MWRs by reducing energy consumption through band sharing algorithm. The contributions of this work are: (i) proposing an Energy Aware Scheduling Algorithm (e-ASA) to improve utilization of the system while decreasing energy consumption in MWRs, (ii) developing energy consumption model for MWRs, and (iii) comparing the performance of single and multi-band Wi-Fi routers in terms of resource usage, throughput and energy consumption. Results show that the proposed method uses system resources efficiently and decreases energy consumption of the multi-band systems up 


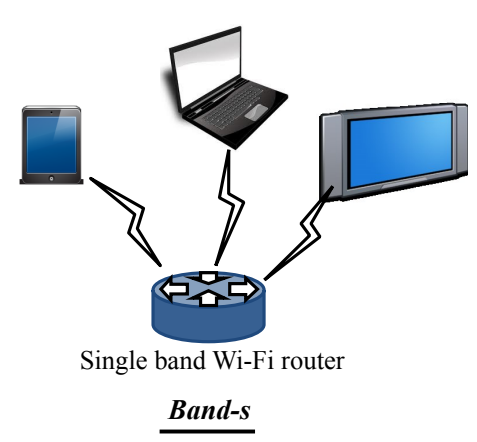

Fig. 1: Single band architecture.

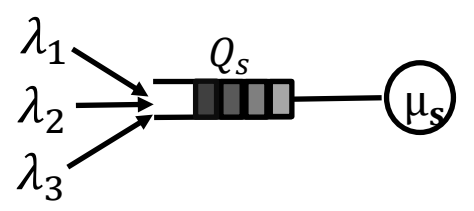

Fig. 4: Single band queuing system.

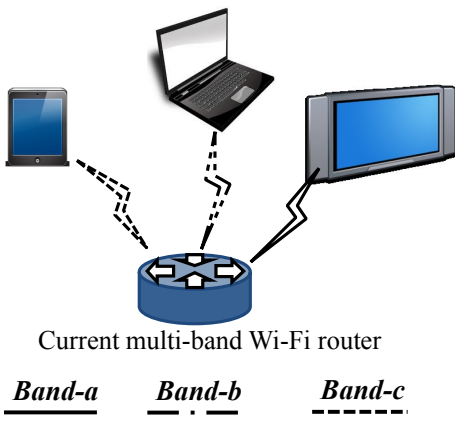

Fig. 2: Current multi-band architecture

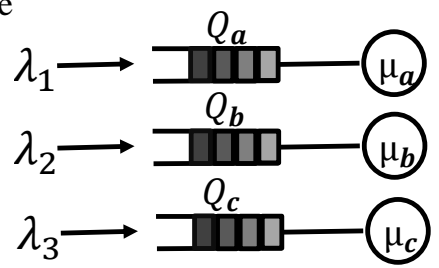

Fig. 5: Current multi-band queuing system.

to $60 \%$ without reducing the system throughput. Our proposed energy aware scheduling algorithm and related analysis will help network engineers build next generation wireless routers by considering the energy usage of the systems.

The rest of the paper is organized as follows. In Section II, we explain the architectures of the single band and multiband Wi-Fi routers, and followed by the proposed scheduling algorithm for multi-shared-band routers in Section III. Section IV presents the simulation environment to analyze the performance of the routers systems. In Section V, we present the simulation results showing the performance in terms of resource usage, energy consumption and throughput. Finally, Section VI has the concluding remarks.

\section{Single And Multi Band Router Architectures}

In this section, the communication scenario and queuing models of single band and mult-band Wi-Fi routers are explained.

\section{A. The Communication Models of Single and Multi Band Routers}

Fig. 1 shows the single band Wi-Fi router architecture. All devices such as tablet, TV and laptop in the network are connected to the router by using only Band-s. Therefore, simultaneously connecting several devices to the router over one band dramatically decreases the quality of the service.

On the other hand, the current multi-band Wi-Fi router uses several bands to send and receive data as presented in Fig. 2. Each device can connect different bands. For example, while the laptop is connecting the router over Band-b, the tablet is using Band-c. Although, simultaneously connecting several devices to the router does not decreases the quality of the service, the utilization of bands are not considered.

Similar to the current multi-band Wi-Fi router, the multishared-band Wi-Fi router can also use a number of bands to communicate with devices as showed in Fig. 3. However, the devices can simultaneously send and receive data over different bands [7], [8]. Therefore, all bands are utilized and the system can have higher throughput.

\section{B. The Queuing Models of Single and Multi Band Routers}

Fig. 4 shows a queuing model sample for single band routers. There is one server with service rate, $\mu_{s}$ and a buffer $\left(Q_{s}\right)$. The incoming traffic with arrival rates, $\lambda_{1}, \lambda_{2}$, and $\lambda_{3}$, is served by one server. When the server is busy and there is an empty space in the buffer, a new arrived request is enqueued to the buffer, $Q_{s}$.

However, multi-band Wi-Fi routers have three servers with service rates $\mu_{a}, \mu_{b}$ and $\mu_{c}$, and buffers $\left(Q_{a}, Q_{b}, Q_{c}\right)$ as showed Figs. 5 and 6. In current MWRs (see Fig. 5), each user traffic is served by only one server. However, multiple servers can simultaneously serve to each user in the multishared-band MWRs (see Fig. 6). Therefore, the band sharing model improves the system performance. However, because of active multi-band operations, the energy consumption of MWRs and devices is significantly increasing. To decrease the energy consumption, we have developed Energy Aware Scheduling Algorithm (e-ASA).

\section{Energy Aware Scheduling Algorithm (e-ASA)}

The energy aware scheduling algorithm is developed to decrease energy consumption of the multi-band system by following wake up and sleep procedures of the bands according to incoming traffic. For example, if there is one user and the user can be satisfied by one band, the router only uses one band for data transfer. We have considered three crucial parameters which are overall arrival rate of data traffic, total service rate of the bands and a threshold to developed e-ASA. 


\section{A. Notations}

Notations in Table I will be used in the rest of the paper.

TABLE I: Notations

\begin{tabular}{lll}
\hline$i$ & $\in$ & $\{s, a, b, c\}$ \\
$Q_{i}$ & $\triangleq \quad$ Queue of $\mu_{i}$ \\
$N_{i}$ & $\triangleq \quad$ Size of $Q_{i}$ \\
$\rho_{i}$ & $\triangleq \lambda_{i} / \mu_{i}$ \\
$\lambda$ & $\triangleq$ & $\sum_{i=1}^{3} \lambda_{i}$ \\
$\mu$ & $\triangleq \mu_{a}+\mu_{b}+\mu_{c}$ \\
$\theta$ & $\triangleq \quad$ Predetermined threshold \\
$D$ & $\triangleq \quad$ Drop probability \\
$\gamma$ & $\triangleq \quad$ Throughput rate \\
$E$ & $\triangleq \quad$ Energy consumption \\
\hline
\end{tabular}

\section{B. Wake Up and Sleep Modes of Bands}

The wake up and sleep operations of one to two bands are processed to save energy in the multi-shared-band architecture. When the bands are not needed, the bands operation are terminated and changed the mode of the bands to sleep mode. If the bands are required because of heavy user loads, the mode of non-active bands is changed to wake up mode and becomes active. Here, it is very important to decide when one, two or three bands are needed because the performance of the system depends on the band operations. Therefore, the expected throughput of the system is measured for three possible cases (one, two, or three bands are active) by using queuing theory in order to efficiently determine the required number of bands. We have used the predetermined threshold $(\theta)$ and the expected throughput of three cases while determining how many bands need to be active. For example, assume that the expected throughput rates are $0.7,0,8$, and 0.9 by using one, two and three bands, respectively and the threshold is 0.8. Therefore, only two bands become active.

The expected throughput of the system for the proposed architecture is measured by using $\mathrm{M} / \mathrm{M} / 1 / \mathrm{N}$ [10]. In order to find the expected throughput, the expected drop rate $(D)$ must be found. From M/M/1/N, drop rate can be written as:

$$
D=\rho^{N} \frac{1-\rho}{1-\rho^{N+1}}
$$

where $\rho=\lambda / \mu$ and $N$ is the queue length. $\mu$ is determined based on service time and bandwidth. In our case, Transmission Time Interval (TTI) is $1 \mathrm{~ms}$ and bandwidth is $20 \mathrm{MHz}$ in all three bands. Therefore, service rates of all three bands are same $\left(\mu_{a}=\mu_{b}=\mu_{c}\right)$ and $\mu$ can be $\mu_{a}, \mu_{a}+\mu_{b}$ or $\mu_{a}+\mu_{b}+\mu_{c}$. $N$ can be 150,75 , or 50 according to number of active bands. Therefore, by using drop rate $(D)$, the throughput rate $(\gamma)$ will be:

$$
\gamma=1-D
$$

\section{The Scheduling Procedure}

The scheduling algorithm e-ASA is processed for users in the multi-shared-band Wi-Fi routers as follow:
- The traffic arrival rate information is determined by the router according to past experiences.

- Partially or fully CQI feedback is obtained to measure the quality of band links. Note that, although CQI is low, the router can transfer only a limited number of packets over that link.

- The required number of bands is determined. It is very important to note that the active bands are also selected based on the coverage. For example, if two bands are enough to serve all users and all users are in the coverage areas of Band- $a$ and Band- $b$ but not in the coverage area of Band-c, then the selected bands to be active are Band-a and Band-b.

- If the communication between users and the router is ensured by non-active bands (which is recently decided to become non-active), the communication links between users and the router are recreated by using new active bands. (Here, firstly the communication links between users and the router is created by using new active bands then the selected bands which is decided to be non-active is processed).

- To increase the efficiency and QoS, packet transferring priority is given to the band, which has the least number of active users (Least Load) and which has the highest CQI. If the band is not enough to data traffic, the other bands can also be used.

\section{Simulation EnViRonment}

A discrete event simulation has been implemented in Matlab by considering the scheduling algorithm and the router architectures which are mentioned in Sections II and III.

\section{A. Assumptions for Wi-Fi Routers}

It is assumed that there are three types of Wi-Fi routers which are single band, current multi-band and the multishared-band routers. While the single band router has only one band, the multi-band routers have three bands to provide service to users. In addition, the routers can only operate over one channel on each band. Some parameters of the routers are summarized in Table II.

\section{TABLE II: Parameters of Wi-Fi Routers}

\begin{tabular}{lll}
\hline Used Bands & $\triangleq$ & $2.4 \mathrm{GHz}, 3.6 \mathrm{GHz}, 5 \mathrm{GHz}$ \\
Length of $Q_{s}$ & $\triangleq$ & 150 packets \\
Length of $Q_{a}, Q_{b}$ and $Q_{c}$ & $\triangleq$ & 50 packets \\
Bandwidth size on each bands & $\triangleq$ & . \\
Modulations & $\triangleq$ & QPSK, 16QAM, and 64QAM \\
Channel Quality Index (CQI) & $\triangleq$ & $3,5,7$, and 11 \\
Transmission Time Interval & $\triangleq$ & $1 \mathrm{~ms}$ \\
Threshold for one band & $\triangleq$ & 0.8 \\
Threshold for two bands & $\triangleq$ & 0.9 \\
$\alpha$ and $\beta$ & $\triangleq$ & 10 and 3, respectively \\
\hline
\end{tabular}

\section{B. Assumptions for Users}

It is assumed that all users can simultaneously connect multiple bands in the system. However, according to router 
architectures, users can transfer data over one or multiple bands. Initially, users are uniformly distributed in the coverage area of the $\mathrm{Wi}-\mathrm{Fi}$ routers and they can move around of the routers in specified time interval. Therefore, CQI can change for each user. Each user can only download or upload one type of traffic. Packet arrivals follow Poisson distribution and packet arrival rates of traffic are enlarged when the number of users is increased. Selected Transmission Time Interval (TTI) for a packet is $1 \mathrm{~ms}$.

\section{Band Selection and Packet Scheduling}

In the simulation, the routers can only operate over one channel from each band. When users arrive, the routers need to assign a band to each user in the current multi-band router. In the single band router, the band selection is not a problem because there is only one band and all users must use same band. However, the current MWR allows each user to transfer data over only one band and the band is automatically assigned to users by selecting least loaded band. For example, if there are 9 users around a current tri-band $\mathrm{Wi}-\mathrm{Fi}$ router, three users are allocated to each band. Therefore, least loaded allocation well balance distribution of the users to bands. On the other hand, the multi-shared-band MWRs, the users can transfer data over all active bands.

In addition to band selection, packet scheduling is needed to obtain results in the simulation. Packet arrival rate from all users are kept same for all test cases. CQI for all users can be one of four options which are given in Table II because of movement of users around the routers. Each packet is transferred by using the assigned band which minimizes packet delay in the multi-shared-band routers. Packet scheduling is first come first serve in single and current multi-band routers because a user only uses one band to send and receive data. If there is no available bands to serve arrived packets, packets are enqueued to corresponding band queues in single and current multi-band routers. However, packets are enqueued to corresponding band queues based on minimum delay measurement in multi-shared-band routers. If there is no spaces in queues, arrived packets are dropped.

\section{Observation Methodology}

The results in Section $\mathrm{V}$ are average of 100 realizations for different number of users. The effect of light and heavy user loads on the energy consumption and the performance of the single band router, current multi-band, multi-shared-band and e-ASA based multi-shared-band are investigated.

We present the performance of single band and multi band (current and multi-shared-band) routers by comparing overall band usage rate, throughput rate and energy consumption. Band usage rate of each band is measured by dividing total packets of active users on each band to total capacity of the bands for each discrete time. Then the average band usage rate is obtained by averaging the measured band usage rate of each band. Throughput rates are measured by dividing transferred packets to all generated packets. Therefore, while the number of users is increased, throughput rate per user decreases because the bands are shared by users. Energy consumption of a router for each band $\left(E_{a}, E_{b}, E_{c}\right.$ for Band-a,Band-b,Band-c) is obtained by using utilization of the bands $\left(\rho_{a}, \rho_{b}, \rho_{c}\right.$ for Band-a, Band-b,Band-c) and simulation time (T) as follows:

$$
E_{a}=T *\left(\rho_{a} * \alpha+\left(1-\rho_{a}\right) * \beta\right)
$$

where $\alpha$ presents the energy consumption during the data transfer and $\beta$ is the energy consumption during the standby. After finding $E_{b}$ and $E_{c}$ similar to $E_{a}$, sum of them gives the overall energy consumption of the router. Therefore, the overall energy consumption of the router $(E)$ will be:

$$
E=E_{a}+E_{b}+E_{c}
$$

As a result of these evaluations, trade off between resource usage, energy efficiency and managed QoS are compared for three types routers.

\section{RESUlts}

In this section, overall performances of single band and multi-band $\mathrm{Wi}-\mathrm{Fi}$ routers are presented. We have also showed the effects of e-ASA on the multi-shared-band router. In Figures, Single is the single band router, Current is the current multi-band router, Shared is the multi-shared-band router without any restriction (all bands are active any time), and $\operatorname{Shared}(e-A S A)$ is e-ASA based multi-shared-band router.

\section{A. Band Usage Rate}

Fig. 7 shows band usage rates of single band and multi-band routers (current, multi-shared-band and e-ASA based multishared-band). Band usage rates of all cases are lower than 0.2 when the number of the users in the system is less than 4 . However, band usage rates of current and multi-shared-band routers slowly increase when the number of users is lower than 8. In contrast to multi-band routers, the band usage rate of the single band router is sharply increasing while the number of users is greater than 4. Because of limited resources in single band routers, the sharp increment points of single, and current and multi-shared-band routers are different. On the other hand, the band usage of e-ASA based multi-shared-band router varies while the number of users is raising because of active number of bands. It is important to note that, the single band router has the highest band usage rate because of limited resources.

\section{B. Energy}

Fig. 8 demonstrates the energy consumption of single and multi-band routers. While the energy consumption of the current and multi-shared-band routers are very close for all number of users, the energy consumption of single and eASA based multi-shared-band routers is equal until the number of the user is 8 . Moreover, the energy consumption of the current and multi-shared-band routers is 2 to 3 times higher than the energy consumption of single and e-ASA based multishared-band routers until the number of the user is 8 . When 


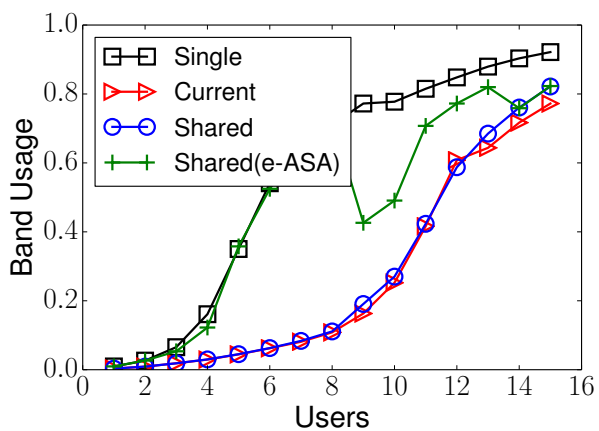

Fig. 7: Overall bands usage rates of the routers.

the number of users is getting larger than 8 , the energy consumption of e-ASA based multi-shared-band routers is sharply increasing and almost reaches to the energy consumption of the current and multi-shared-band routers. This is because of dynamic arrangement of active bands of e-ASA. Furthermore, the active number of bands in e-ASA based multi-shared-band router is becoming 3 when the number of users reaches 14 . Therefore, the energy consumption of e-ASA based multishared-band is passing the energy consumption of the current multi-band routers and reaches the energy consumption of the multi-shared-band router.

\section{Throughput}

Fig. 9 depicts throughput rates of single and multi-band routers. Until the number of users reaches 8 , all routers are capable of serving to all traffic of users. However, the throughput rate of the single band router is regularly decreasing while the number of users is getting higher. On the other hand, the throughput rate of the current multi-band router slowly decreases after the number of users is 10 . In contrast to single and current multi-band routers, the throughput rates of multishared-band and e-ASA based multi-shared-band routers are still optimum while the number of users is 15 . However, when the number of users is raising, the throughput rates of the multi-shared-band and e-ASA based multi-shared-band routers will also decrease.

It is important to note that, when the number of the users are 8 or 13 , there is insignificant amount of decrease in throughput rate of e-ASA based multi-shared-band router because e-ASA allows only one band to be active until the number of the users is 8 and two bands when the number of the users is between 9 and 13. After that, all bands are activated by e-ASA.

\section{Summary of Results}

Based on the results, we make the following observations: (i) the throughput rate of multi-band Wi-Fi routers is significantly higher than the throughput rate of single band WiFi routers, (ii) the energy consumption of current and multishared-band routers are close to each other, and (iii) e-ASA can decrease energy consumption of the multi-shared-band up to $60 \%$ without decreasing the throughput rate of the system when the system is under light users loads.

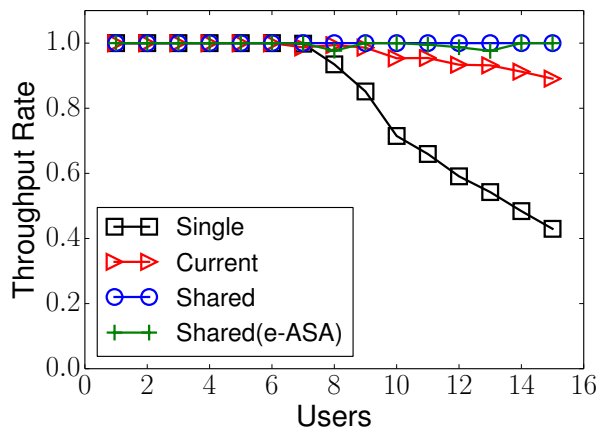

Fig. 9: Throughput rates of the routers.

\section{CONCLUSION}

In this paper, we have proposed an energy aware scheduling algorithm for next generation Wi-Fi routers by considering traffic arrivals, channel quality indicator of bands, and service rates. Band usage, energy consumption and throughput rate of single band, current multi-band and multi-shared-band Wi-Fi routers have been demonstrated through an extensive simulation. Results show that Energy Aware Scheduling Algorithm uses system resources efficiently and decreases energy consumption of the multi-band routers up to $60 \%$. Our proposed energy aware scheduling algorithm and related analysis will help network engineers build next generation wireless routers by considering the energy usage of the systems.

\section{REFERENCES}

[1] H. Singh, J. Hsu, L. Verma, S. S. Lee, and C. Ngo, "Green operation of multi-band wireless LAN in $60 \mathrm{GHz}$ and $2.4 / 5 \mathrm{GHz}$," in Consumer Communications and Networking Conference $(C C N C)$, Las Vegas, NV, Jan 9-12, 2011, pp. 787-792.

[2] E. Perahia, C. Cordeiro, M. Park, and L. L. Yang, "IEEE 802.11ad: Defining the next generation multi-Gbps Wi-Fi," in 7th IEEE Consumer Communications and Networking Conference (CCNC), Las Vegas, NV, Jan 9-12, 2010.

[3] I. F. Akyildiz, D. M. Gutierrez-Estevez, and E. C. Reyes, "The evolution to 4G cellular systems: LTE-Advanced," Physical Communication, vol. 3, pp. 217-244, Mar 2010.

[4] S. Zeadally, S. U. Khan, and N. Chilamkurti, "Energy-efficient networking: past, present, and future," Journal of Supercomputing, vol. 62, pp. 1093-1118, Dec 2012.

[5] S. Singh, R. Mudumbai, and U. Madhow, "Distributed coordination with deaf neighbors: Efficient medium access for $60 \mathrm{GHz}$ mesh networks," in IEEE INFOCOM, San Diego, CA, Mar 14-19, 2010.

[6] L. Verma and S. S. Lee, "Multi-band Wi-Fi systems: A new direction in personal and community connectivity," in IEEE International Conference on Consumer Electronics (ICCE), Las Vegas, NV, Jan 9-12, 2011, pp. 665-666.

[7] M. S. Hossain, H. Narman, and M. Atiquzzaman, "A novel scheduling and queue management scheme for multi-band mobile routers," in IEEE International Conference on Communications (ICC), Budapest, Hungary, June 9-13, 2013, pp. 3787 - 3791.

[8] H. Narman, M. S. Hossain, and M. Atiquzzaman, "Multi class traffic analysis of single and multi-band queuing system," in IEEE Global Communications Conference (GLOBECOM), Atlanta, GA, Dec 9-13, 2013, pp. $1422-1427$.

[9] N. Blackmore. (2014, July) Wireless internet router is expensive on standby but is it wise to turn off at night? Accessed: Dec. 4, 2014. [Online]. Available: http://www. telegraph.co.uk/finance/personalfinance/money-saving-tips/10953517/ Wireless-internet-router-is-expensive-on-standby-but-is-it-wise-to-turn-/ off-at-night.html

[10] D. Gross and C. M. Harris, Fundamentals of Queueing Theory (Wiley Series in Probability and Statistics). Wiley-Interscience, Feb 1998. 